\title{
Assessment of Quad-Frequency Long-Baseline Positioning with BeiDou-3 and Galileo Observations
}

\author{
Liwei Liu ${ }^{1,2}$, Shuguo Pan ${ }^{1,2, *}$, Wang Gao ${ }^{1,2}$, Chun Ma ${ }^{1} \mathbb{C}$, Ju Tao ${ }^{3}$ and Qing Zhao ${ }^{4}$ \\ 1 School of Instrument Science and Engineering, Southeast University, Nanjing 210096, China; \\ liuliwei@seu.edu.cn (L.L.); gaow@seu.edu.cn (W.G.); machun@seu.edu.cn (C.M.) \\ 2 Key Laboratory of Micro-Inertial Instrument and Advanced Navigation Technology, Southeast University, \\ Nanjing 210096, China \\ 3 Xinxing Jihua Science and Technology Development CO., LTD., 9th Floor, Building 6, District 15, No. 188, \\ South Fourth Ring West Road, Fengtai District, Beijing 100070, China; ceratj@163.com \\ 4 School of Transportation, Southeast University, Nanjing 210096, China; zhaoqing@seu.edu.cn \\ * Correspondence: psg@seu.edu.cn
}

check for

updates

Citation: Liu, L.; Pan, S.; Gao, W.; Ma, C.; Tao, J.; Zhao, Q. Assessment of Quad-Frequency Long-Baseline Positioning with BeiDou-3 and Galileo Observations. Remote Sens. 2021, 13, 1551. https://doi.org/ $10.3390 /$ rs13081551

Received: 26 February 2021

Accepted: 13 April 2021

Published: 16 April 2021

Publisher's Note: MDPI stays neutral with regard to jurisdictional claims in published maps and institutional affiliations.

Copyright: (c) 2021 by the authors. Licensee MDPI, Basel, Switzerland. This article is an open access article distributed under the terms and conditions of the Creative Commons Attribution (CC BY) license (https:// creativecommons.org/licenses/by/ $4.0 /)$.

\begin{abstract}
Quad-frequency signals have thus far been available for all satellites of BeiDou-3 and Galileo systems. The major benefit of quad-frequency signals is that more extra-wide-lane (EWL) combinations can be formed with quad-frequency than with triple- or dual-frequency, of which the ambiguities can be fixed instantaneously in medium and long baselines. In this paper, the longbaseline positioning algorithm based on optimal triple-frequency EWL/wide-lane (WL) combinations of BeiDou-3 and Galileo is proposed. First, the theoretical precision of multi-frequency combinations of BeiDou-3 and Galileo is studied, and EWL/WL combinations with a small noise amplitude factor and a small ionospheric scalar factor are selected. Then, geometry-free methods are used to estimate the a priori precision of EWL/second EWL/WL signals for different combination schemes. Second, the double-differenced (DD) geometry-based function models of quad-frequency configurations and three different triple-frequency configurations are given, and the DD ionospheric delays are estimated as unknown parameters. In the end, the real BeiDou- 3 and Galileo data are used to evaluate the positioning preference. The results show that, when using fixed EWL observations to constrain WL ambiguities, the proposed triple-frequency EWL/WL signals composed of (B1I,B3I,B2a) of BeiDou-3 and (E1,E5a,E6) of Galileo can achieve the same precision as the quad-frequency signals. Therefore, the method proposed in this article can realize long-baseline instantaneous decimeter-level positioning while reducing the dimension of matrix and improving calculation efficiency.
\end{abstract}

Keywords: BeiDou-3; Galileo; quad-frequency observations; long baseline; real-time kinematic (RTK); geometry-based (GB)

\section{Introduction}

Currently, Global Navigation Satellite Systems (GNSSs) are developing towards multisystems and multi-frequencies. BeiDou Navigation Satellite System (BDS) completed its global satellite network on 31 July 2020. Among them, 30 BDS-3 satellites provide four public service signals-B1I, B1C, B2a, and B3I-in three frequency bands-B1, B2, and B3. The European system Galileo Satellite Navigation System is a multi-frequency global satellite navigation system that was independently developed by Europe and not controlled by the military. As of June 2020, Galileo has launched 26 satellites, of which 24 are in orbit, 2 in orbit verification (IOV) satellites and 22 full operational capability (FOC) satellites, and is close to achieving global deployment [1,2]. With the combination of BDS-3 and Galileo, the number of visible satellites increases. Therefore, some scholars have also studied the positioning performance of GPS, BDS-2, and BDS-3 or Galileo data fusion. Miao et al. combined BDS-2 and BDS-3 and evaluated their positioning performance [3]. Wang et al. also analyzed the positioning performance of the combination of Galileo and BDS-2 [4]. 
The combined use of these satellite constellations can improve the reliability, accuracy, and stability of GNSS positioning performance [5]. However, previous studies only used dual-frequency or triple-frequency data. Zhang et al. analyzed the triple-frequency, quadfrequency, and penta-frequency linear combinations provided by BDS-3. The number of high-quality signals of quad-frequency is about ten times that of triple-frequency [6]. Wang et al. used precise ephemeris and Galileo geometry-based data after multipath correction to analyze the ambiguity resolution (AR) performance of known baselines. The empirical success rate of combinations after multipath correction increases by $4-5$ percentage points in the case of five frequencies, but it needs a multipath calculation at least one week in advance and cannot be applied to real-time calculations [7]. Tu et al. analyzed the shortbaseline positioning experiment of the multi-frequency observation data from the Galileo system. Among them, the quad-frequency positioning performance is poor due to the bad observation quality of the E1 frequency, and the positioning precision of quad-frequency is not as good as that of triple-frequency. In addition, the fourth frequency used in the experiment is E5 instead of E6 [1].

The traditional short-baseline RTK can also resolve ambiguities in a single epoch and can obtain centimeter-level positioning accuracy [8]. However, when the operating range increases, the correlation of the spatial error gradually weakens and the positioning performance deteriorates. Therefore, in order to ensure a wide range of high-precision and rapid positioning, a dense reference station network is required. On the contrary, precise point positioning (PPP) can also provide centimeter-level positioning performance and does not require reference stations, but its major limitations are a slow convergence speed and a long initialization time [9-11]. Even with multiple GNSSs, PPP still takes about $10 \mathrm{~min}$ to obtain reliable integer ambiguity resolutions. In addition, if an open sky-view is not constantly available, frequent re-convergences can destroy the practicability of real-time PPP completely [12,13].

Multi-frequency signals provide opportunities for high-precision and rapid positioning. Generally, multi-frequency signals can increase the number of combinations [14] and provide additional long-wavelength, small ionospheric scalar factor, and easy-to-fix carrier phase combinations. Moreover, it will reduce location-blind areas (such as special environments) [15] and weaken the influence of various error sources on the combined ambiguity [16,17] to apply the long-baseline RTK [18] and large-scale network RTK [19].

In general, it is easier to solve the ambiguities of EWL/WL based on the observation information of multi-frequency and multi-system, so it is equivalent to adding higherprecision 'pseudo-range' observations than dual-frequency observations. Based on these advantages, it is conducive to construct a multi-scale positioning model that can meet different scenarios to better meet the diversified and differentiated needs of modern surveying and emerging location service industries for high-precision positioning [20].

In this study, we try to make full use of all BDS-3 and Galileo quad-frequency data to improve the performance of high-precision and rapid long-baseline positioning. Then, we try to find out the frequency point that can be eliminated in the quad-frequency signals, which means that we select three frequencies to obtain the theoretically equivalent positioning precision with quad-frequency signals. In this way, the dimension of matrix is reduced and the calculation efficiency is also improved to make preparations for adding new satellite navigation systems in the future.

The structure of this paper is as follows. In Section 2, the multi-frequency single-epoch DD mathematical model of BDS-3 and Galileo is derived. In Section 3, satellite availability and RDOP values are analyzed and positioning performance and EWL/Second EWL/WL ambiguity fractions in the four combination schemes are analyzed. In Section 4, the success rate of theoretical EWL AR with different total errors is discussed. Some conclusions are given in Section 5. 


\section{Materials and Methods}

In this section, we describe the quad-frequency DD observation equations and stochastic model. We analyzed the theoretical a priori precision of quad-frequency and three different triple-frequency EWL/WL combinations in BDS-3 and Galileo. Four publicly available signals of BDS-3 and Galileo are shown in Table 1. The order number in the second column is a simplification of the two system signals, $f_{1}, f_{2}, f_{3}, f_{4}$ correspond to B1C, B1I, B3I, and B2a for BDS-3 and to E1, E5a, E5b, and E6 for Galileo, respectively.

Table 1. Quad-frequency signals of BDS-3 and Galileo.

\begin{tabular}{cccc}
\hline Satellite Systems & Order Number & Signals & Frequencies (MHz) \\
\hline BDS-3 & 1 & B1C & 1575.420 \\
& 2 & B1I & 1561.098 \\
3 & B3I & 1268.520 \\
& 4 & B2a & 1176.450 \\
Galileo & 1 & E1 & 1575.420 \\
& 2 & E5a & 1176.450 \\
& 3 & E5b & 1207.140 \\
& 4 & E6 & 1278.750 \\
\hline
\end{tabular}

It can be seen that the center frequency of the B1C and B2a signals of BDS-3 are the same as the center frequency of the Galileo E1 and E5a signals. Therefore, some combinations with the same wavelength can be formed from the two systems.

\subsection{Double-Differenced BDS-3 and Galileo Mathematical Model}

The basic DD observation equations of pseudo-range and carrier phase measurements for a system 'sys' (denotes BDS-3 and Galileo) and frequency band $i(i=1,2,3,4)$ can be expressed as follows:

$$
\left\{\begin{array}{c}
\Delta \nabla P_{i}^{\text {sys }}=\Delta \nabla \rho+\eta_{i}^{\text {sys }} \Delta \nabla I_{1}+\Delta \nabla T+\Delta \nabla \varepsilon_{P} \\
\Delta \nabla \phi_{i}^{\text {sys }}=\Delta \nabla \rho-\eta_{i}^{\text {sys }} \Delta \nabla I_{1}+\Delta \nabla T+\lambda_{i}^{\text {sys }} \Delta \nabla N_{i}^{\text {sys }}+\Delta \nabla \varepsilon_{\phi}
\end{array}\right.
$$

where the symbol ' $\Delta \nabla^{\prime}$ ' represents the DD operator; $P_{i}^{\text {sys }}$ and $\phi_{i}^{\text {sys }}$ represent the pseudorange and carrier phase observation values of the corresponding system, respectively; $\rho$ is the geometric distance between a satellite and a receiver; $I_{1}$ is the first-order ionospheric delay at the first frequency; $\eta_{i}^{\text {sys }}$ is the first-order ionospheric scalar factor of the corresponding system; $T$ is the tropospheric delay; $\varepsilon_{P}$ and $\varepsilon_{\phi}$ are the measurement noise of the pseudo-range and carrier phase, respectively; and $N_{i}^{s y s}$ is the DD ambiguity of the corresponding system.

The corresponding linearized DD pseudo-range and carrier phase measurement equations can be expressed as follows:

$$
\left\{\begin{array}{c}
\Delta \nabla P_{i}^{\text {sys }}=A X+\Delta \nabla \varepsilon_{P} \\
\Delta \nabla \phi_{i}^{\text {sys }}=A X+\lambda_{i}^{\text {sys }} \Delta \nabla N_{i}^{\text {sys }}+\Delta \nabla \varepsilon_{\phi}
\end{array}\right.
$$

where $A$ is the linear coefficient matrix and $X$ denotes the baseline parameters and atmospheric parameters.

Omitting the system superscript, the combined DD observation equations of quadfrequency signals can be written as follows [16]:

$$
\Delta \nabla \phi_{(i, j, k, m)}=\Delta \nabla \rho-\eta_{(i, j, k, m)} \Delta \nabla I_{1}+\Delta \nabla T+\lambda_{(i, j, k, m)} \Delta \nabla N_{(i, j, k, m)}+\Delta \nabla \varepsilon_{\phi(i, j, k, m)}
$$

where

$$
\Delta \nabla \phi_{(i, j, k, m)}=\frac{i \cdot f_{1} \cdot \Delta \nabla \phi_{1}+j \cdot f_{2} \cdot \Delta \nabla \phi_{2}+k \cdot f_{3} \cdot \Delta \nabla \phi_{3}+m \cdot f_{4} \cdot \Delta \nabla \phi_{4}}{i \cdot f_{1}+j \cdot f_{2}+k \cdot f_{3}+m \cdot f_{4}}
$$




$$
\begin{aligned}
\eta_{(i, j, k, m)} & =\frac{f_{1}^{2}\left(\frac{i}{f_{1}}+\frac{j}{f_{2}}+\frac{k}{f_{3}}+\frac{m}{f_{4}}\right)}{i \cdot f_{1}+j \cdot f_{2}+k \cdot f_{3}+m \cdot f_{4}} \\
\lambda_{(i, j, k, m)} & =\frac{c}{i \cdot f_{1}+j \cdot f_{2}+k \cdot f_{3}+m \cdot f_{4}}
\end{aligned}
$$

where the coefficients $i, j, k, m$ of the combinations are represented by $i_{1}, j_{1}, k_{1}, m_{1}$ and $i_{2}, j_{2}, k_{2}, m_{2}$ in BDS-3 and Galileo, respectively; $\Delta \nabla \phi_{(i, j, k, m)}$ is a DD carrier phase observation of the combined signal; the expression of $\Delta \nabla P_{(i, j, k, m)}$ is similar to $\Delta \nabla \phi_{(i, j, k, m)}$; $\eta_{(i, j, k, m)}$ is the ionospheric scalar factor of the combined signal; $\lambda_{(i, j, k, m)}$ is the wavelength of the combined signal; and $c$ is the velocity of light.

\subsection{Determination of the EWL Ambiguity}

Taking BDS-3 as an example, the EWL carrier phase measurements and the pseudorange code measurements on B1C, B1I, B3I, and B2a can be used to form a set of observation equations, expressed as follows:

$$
\left[\begin{array}{c}
\boldsymbol{v}_{P_{1}} \\
\boldsymbol{v}_{P_{2}} \\
\boldsymbol{v}_{P_{3}} \\
\boldsymbol{v}_{P_{4}} \\
\boldsymbol{v}_{E W L}
\end{array}\right]=\left[\begin{array}{ccc}
\boldsymbol{B} & \eta_{1} \boldsymbol{I}_{S} & 0 \\
\boldsymbol{B} & \eta_{2} \boldsymbol{I}_{S} & 0 \\
\boldsymbol{B} & \eta_{3} \boldsymbol{I}_{S} & 0 \\
\boldsymbol{B} & \eta_{4} \boldsymbol{I}_{S} & 0 \\
\boldsymbol{B} & -\eta_{E W L} \boldsymbol{I}_{S} & \lambda_{E W L} \boldsymbol{I}_{S}
\end{array}\right]\left[\begin{array}{c}
\boldsymbol{x} \\
\boldsymbol{i o n} \\
\boldsymbol{N}_{E W L}
\end{array}\right]-\left[\begin{array}{c}
\boldsymbol{l}_{P_{1}} \\
\boldsymbol{l}_{P_{2}} \\
\boldsymbol{l}_{P_{3}} \\
\boldsymbol{l}_{P_{4}} \\
\boldsymbol{l}_{E W L}
\end{array}\right]
$$

where the symbols $v_{P_{1}}, v_{P_{2}}, v_{P_{3}}, v_{P_{4}}$ represent the DD pseudo-range observation residual vectors on B1C, B1I, B3I, and B2a, respectively; $v_{E W L}$ represents the residual vector of DD carrier phase observations of an EWL signal; $\boldsymbol{B}$ is the design matrix of the baseline parameter $x ; \eta_{E W L}$ is the ionospheric scalar factor of an EWL signal; $s$ represents the number of DD satellite pairs tracked in a current epoch; $\boldsymbol{I}_{S}$ is an identity matrix with dimension $s$; ion and $N_{E W L}$ represent an unknown DD ionospheric bias matrix and a DD EWL ambiguities matrix with dimension $s$, respectively; and the last terms $\boldsymbol{l}_{P_{1}}, \boldsymbol{l}_{P_{2}}, \boldsymbol{l}_{P_{3}}, \boldsymbol{l}_{P_{4}}$, and $\boldsymbol{l}_{E W L}$ are observed minus the computed (OMC) vectors from their corresponding observations.

Suppose that the combination coefficients of EWL are $i^{\prime}, j^{\prime}, k^{\prime}, m^{\prime}$, the covariance matrix of (9) can then be written as follows [21]:

$$
\begin{gathered}
\boldsymbol{Q}_{D D}=\boldsymbol{T}\left(2 \operatorname{diag}\left(\left[\sigma_{P}^{2}, \sigma_{\phi}^{2}\right]\right) \otimes \boldsymbol{I}_{4} \otimes \boldsymbol{P}^{-1}\right) \boldsymbol{T}^{T} \\
\boldsymbol{T}=\left[\begin{array}{cc}
\boldsymbol{I}_{4 \cdot s} & \mathbf{0} \\
\mathbf{0} & \boldsymbol{T}_{E W L} \otimes \boldsymbol{I}_{S}
\end{array}\right] \\
\boldsymbol{T}_{E W L}=\left[i^{\prime}, j^{\prime}, k^{\prime}, m^{\prime}\right]
\end{gathered}
$$

where $\sigma_{P}^{2}$ and $\sigma_{\phi}^{2}$ are the basic variances between undifferenced pseudo-range and carrier phase observations in the zenith direction, respectively; $\otimes$ denotes the Kronecker product; $\boldsymbol{P}=Q^{-1}$ is the DD weight matrix; $Q$ is the cofactor matrix that depends on the elevation of observations [22]; $T$ is a linear transformation matrix used to transform the original observation matrix into a combined observation matrix; and $\boldsymbol{T}_{E W L}$ is a combination coefficient matrix of EWL. It is worth noting that, when calculating the long-baseline, Equation (8) needs to calculate the inter-satellite difference of the two stations separately and to then add them to obtain $Q_{D D}$.

\subsection{Determination of the Second EWL/WL Ambiguity}

Since the wavelength of EWL is long enough, the float ambiguities obtained by the least square method can be fixed by rounding directly. The addition of the EWL carrier phase observations and the fixed ambiguities can be regarded as accurate pseudo-range observations [23]. Then, the fixed EWL combinations are used as known observations to 
estimate second EWL (for quad-frequency) or WL combinations, and the corresponding equations are as follows (taking WL as an example):

$$
\left[\begin{array}{c}
\boldsymbol{v}_{E W L}^{\prime} \\
\boldsymbol{v}_{W L}
\end{array}\right]=\left[\begin{array}{ccc}
\boldsymbol{B} & -\eta_{E W L} \boldsymbol{I}_{S} & \mathbf{0} \\
\boldsymbol{B} & -\eta_{W L} \boldsymbol{I}_{S} & \lambda_{W L} \boldsymbol{I}_{S}
\end{array}\right]\left[\begin{array}{c}
\boldsymbol{x} \\
\boldsymbol{i o n} \\
\boldsymbol{N}_{W L}
\end{array}\right]-\left[\begin{array}{c}
\boldsymbol{l}_{E W L}^{\prime} \\
\boldsymbol{l}_{W L}
\end{array}\right]
$$

where $v_{E W L}^{\prime}$ and $v_{W L}$ represent the residual vector of DD pseudo-range observations of precise EWL and DD WL observations, respectively; $-\eta_{W L}$ is the ionospheric scalar factor of the WL signal; and $\boldsymbol{l}_{E W L}^{\prime}$ and $\boldsymbol{l}_{W L}$ are OMC vectors of the corresponding observations, respectively. The ambiguities of $W L$ can be determined by the method of Least-squares AMBiguity Decorrelation Adjustment (LAMBDA) [24]. When quad-frequency is used, the WL of Equation (10) is changed to a second EWL. The wavelength of a second EWL is shorter than the first EWL, which can be fixed using least squares or the LAMBDA method. Then, fixed second EWL observations are used to constrain WL ambiguities.

\subsection{Theoretical Precision of Triple- and Quad-Frequency EWL/WL Signals of BDS-3 and Galileo}

Then, we analyzed the theoretical precision of the instantaneous ambiguity and range parameters at each step in the DD case.

The precision of the GNSS positioning performance depends on the measurement noise of the observations and the strength of the geometric figures between the receivers and the GNSS satellites. The dilution of precision (DOP) factor is often used in positioning to quantitatively reflect the strength of the geometric figures [25]. The calculation of the DOP value and the theoretical precision of the baseline can be expressed as follows [26,27]:

$$
\begin{aligned}
& D O P=\sqrt{\operatorname{trace}\left(\boldsymbol{B}_{a}^{T} \boldsymbol{P} \boldsymbol{B}_{a}\right)^{-1}} \\
& \sigma_{3 D}=\sigma_{b} \times D O P
\end{aligned}
$$

where $\boldsymbol{B}_{a}$ is the design matrix of the entire equations; $\sigma_{3 D}$ is the three-dimensional (3D) theoretical precision of the baseline parameters (DD range); and $\sigma_{b}$ is the normalized baseline noise variance, which is related to the noise of EWL/WL combinations. When the DOP (PDOP for absolute positioning and RDOP for relative positioning) for all satellites in view is equal to 1 , the theoretical precision of station-satellite distance or baseline can be obtained [28]. Therefore, assuming that RDOP is equal to 1 , the theoretical baseline precision can be calculated using Equation (11).

Empirically, the DD pseudo-range and carrier phase precision are set as $50 \mathrm{~cm}$ and $5 \mathrm{~mm}$, respectively. Various combinations can be formed based on the original observations. When the number of frequency increases, the number of optional combined signals also greatly increases, but most of them are unsuitable signals.

Assuming that the coefficient value $i, j, k, m$ ranges from -10 to 10 , some linearly independent EWL, second EWL, and WL combinations with a small noise amplitude factor and a small ionospheric scalar factor can be obtained in the two systems, respectively. Within this range, we first chose the combination with minimal theoretical DD ambiguity standard deviation (STD) as the first EWL, corresponding to the combination of BDS-3 $(1,-1,0,0)$ and Galileo $(0,-1,1,0)$, and the corresponding EWL ambiguity precisions were 0.041 cycles and 0.043 cycles (shown in Tables 2 and 3), respectively. The first choice of second EWL combination was $\frac{\lambda_{E W L}}{2.0}>\lambda_{S E W L}>2.93 \mathrm{~m}$, and then, the combination with the minimum DD ambiguity STD within this wavelength range was selected. Ignoring the troposphere residuals and the orbital residuals, Tables 2 and 3 show the theoretical parameter precisions of BDS-3 and Galileo when the RDOP value is 1, respectively. When no ambiguities were fixed, the precision of the baseline parameter was decided by the DD pseudo-range noise. 
Table 2. Precision of parameters in the BDS-3 quad-frequency (B1C, B1I, B3I, and B2a) DD case.

\begin{tabular}{cccccc}
\hline \multirow{2}{*}{ Parameters } & Wavelength/m & \multicolumn{4}{c}{ Number of Fixed Ambiguities } \\
\cline { 3 - 6 } & & $\mathbf{0}$ & $\mathbf{1}$ & $\mathbf{2}$ & $\mathbf{3}$ \\
\hline B1C-B1I/(cycle) & 20.93 & 0.041 & 0 & 0 & 0 \\
B3I-B2a/(cycle) & 3.26 & 0.110 & 0.110 & 0 & 0 \\
B1I-B2a/(cycle) & 0.78 & 0.334 & 0.314 & 0.209 & 0 \\
DD Range/m & N/A & 1.012 & 0.949 & 0.816 & 0.333 \\
\hline
\end{tabular}

Table 3. Precision of parameters in the Galileo quad-frequency (E1, E5a, E5b, and E6) DD case.

\begin{tabular}{cccccc}
\hline \multirow{2}{*}{ Parameters } & Wavelength/m & \multicolumn{4}{c}{ Number of Fixed Ambiguities } \\
\cline { 3 - 6 } & & $\mathbf{0}$ & $\mathbf{1}$ & $\mathbf{2}$ & $\mathbf{3}$ \\
\hline E5a-E5b/(cycle) & 9.77 & 0.043 & 0 & 0 & 0 \\
E5b-E6/(cycle) & 4.19 & 0.070 & 0.060 & 0 & 0 \\
E1-E5a/(cycle) & 0.75 & 0.379 & 0.358 & 0.298 & 0 \\
DD Range/m & N/A & 1.249 & 1.173 & 1.169 & 0.316 \\
\hline
\end{tabular}

For the function model shown in Equations (7) and (10), when only observation noise is considered, the float ambiguities rounding success rates can be computed as follows [29]:

$$
P(-0.5<x<0.5)=\int_{-0.5}^{0.5} \frac{1}{\sigma \sqrt{2 \pi}} \exp \left(-\frac{x^{2}}{2 \sigma^{2}}\right) d x
$$

where $\sigma$ is the random bias caused by observation noises.

It can be seen that the theoretical precisions of BDS-3 and Galileo are $1.012 \mathrm{~m}$ and $1.249 \mathrm{~m}$, respectively. When only pseudo-range is used, DD range is more than $1.0 \mathrm{~m}$ and decimeter-level instantaneous positioning cannot be obtained. Additionally, after fixing the EWL, the DD range theoretical precisions of BDS-3 and Galileo are $0.949 \mathrm{~m}$ and $1.173 \mathrm{~m}$, respectively, which have slight improvement. Similarly, if the second EWL is directly constrained by pseudo-ranges, the ambiguity STDs of BDS-3 and Galileo are 0.110 cycles and 0.070 cycles, respectively, and the a priori success rates are both greater than $99.9 \%$, so they can be fixed reliably by rounding. In addition, it can be seen that using the fixed EWL observations as accurate pseudo-range observations to constrain second EWL ambiguities cannot greatly improve the precision of second EWL ambiguities. Moreover, the DD range is not improved by much after fixing the second EWL ambiguities compared with EWL, especially for Galileo. However, no matter for BDS-3 or Galileo, the precision of WL ambiguities has the same characteristics: the fixed EWL observations have little restriction on WL ambiguities, and the WL ambiguity STDs of BDS-3 and Galileo are 0.314 cycles and 0.358 cycles, respectively. Compared to without EWL constraints, the a priori success rate only increased by 2.3 and 2.5 percentage points. Nevertheless, the fixed second EWL can significantly improve the precision of WL ambiguities.

After using the fixed second EWL constraints, the WL ambiguity STDs of BDS-3 and Galileo are 0.209 cycles and 0.298 cycles, respectively, and the a priori success rates are $98.3 \%$ and $90.7 \%$, respectively. Compared with the fixed EWL, it has been increased by 9.5 and 6.9 percentage points, respectively. Correspondingly, the quad-frequency WL AR of BDS-3 and Galileo in Section 2.3 can be expressed as follows:

$$
\left[\begin{array}{c}
\boldsymbol{v}_{P}^{C} \\
\boldsymbol{v}_{E W L}^{C} \\
\boldsymbol{v}_{S E W L}^{C} \\
\boldsymbol{v}_{W L}^{C} \\
\boldsymbol{v}_{P}^{E} \\
\boldsymbol{v}_{E W L}^{E} \\
\boldsymbol{v}_{S E W L}^{E} \\
\boldsymbol{v}_{W L}^{E}
\end{array}\right]=\left[\begin{array}{ccccc}
\boldsymbol{e}_{4} \otimes \boldsymbol{B}^{C} & \eta_{P}^{C} \otimes \boldsymbol{I}_{c} & \mathbf{0} & \mathbf{0} & \mathbf{0} \\
\boldsymbol{B}^{C} & -\eta_{E W L} \boldsymbol{I}_{\mathcal{C}} & \mathbf{0} & \mathbf{0} & \mathbf{0} \\
\boldsymbol{B}^{C} & -\eta_{S E W L} \boldsymbol{I}_{\mathcal{C}} & \mathbf{0} & \mathbf{0} & \mathbf{0} \\
\boldsymbol{B}^{C} & -\eta_{W L} \boldsymbol{I}_{\mathcal{C}} & \lambda_{W L}^{C} \boldsymbol{I}_{\mathcal{C}} & \mathbf{0} & \mathbf{0} \\
\boldsymbol{e}_{4} \otimes \boldsymbol{B}^{E} & \mathbf{0} & \mathbf{0} & \boldsymbol{\eta}_{P}^{E} \otimes \boldsymbol{I}_{e} & \mathbf{0} \\
\boldsymbol{B}^{E} & \mathbf{0} & \mathbf{0} & -\eta_{E W L} \boldsymbol{I}_{e} & \mathbf{0} \\
\boldsymbol{B}^{E} & \mathbf{0} & \mathbf{0} & -\eta_{S E W L} \boldsymbol{I}_{e} & \mathbf{0} \\
\boldsymbol{B}^{E} & \mathbf{0} & \mathbf{0} & -\eta_{W L} \boldsymbol{I}_{e} & \lambda_{W L}^{E} \boldsymbol{I}_{e}
\end{array}\right]\left[\begin{array}{c}
\boldsymbol{x} \\
\boldsymbol{i o \boldsymbol { n } ^ { C }} \\
\boldsymbol{N}_{W L}^{C} \\
\boldsymbol{i o n} \boldsymbol{n}^{E} \\
\boldsymbol{N}_{W L}^{E}
\end{array}\right]-\left[\begin{array}{c}
\boldsymbol{l}_{P}^{C} \\
\boldsymbol{l}_{E W L}^{C} \\
\boldsymbol{l}_{S E W L}^{C} \\
\boldsymbol{l}_{W L}^{C} \\
\boldsymbol{l}_{P}^{E} \\
\boldsymbol{l}_{E W L}^{E} \\
\boldsymbol{l}_{S E W L}^{E} \\
\boldsymbol{l}_{W L}^{E}
\end{array}\right]
$$


where $\boldsymbol{v}_{P}^{C}=\left[\boldsymbol{v}_{P_{1}}^{C^{T}}, \boldsymbol{v}_{P_{2}}^{C^{T}}, \boldsymbol{v}_{P_{3}}^{C^{T}}, \boldsymbol{v}_{P_{4}}^{C^{T}}\right]^{T} ; \boldsymbol{l}_{P}^{C}=\left[\boldsymbol{l}_{P_{1}}^{C^{T}}, \boldsymbol{l}_{P_{2}}^{C^{T}}, \boldsymbol{l}_{P_{3}}^{C^{T}}, \boldsymbol{l}_{P_{4}}^{C^{T}}\right]^{T} ; \boldsymbol{v}_{P}^{E}$ and $\boldsymbol{l}_{P}^{E}$ have the same expression; the superscript capitals ' $C$ ' and ' $E$ ' indicate BDS-3 and Galileo, respectively; $\boldsymbol{e}_{n}$ is a column matrix vector with $\mathrm{n}$ rows of elements, all being 1 ; and the subscripts ' $c$ ' and ' $e$ ' of the identity matrix are the dimensions of matrix, corresponding to the number of satellite pairs of BDS-3 and Galileo, respectively. It should be noted that the first two steps of the EWL and second EWL AR processes cannot be ignored.

In the above process, some suboptimal second EWL combinations have been eliminated, for example, the Galileo signal with a wavelength of $2.91 \mathrm{~m}$. However, no suboptimal second EWL signal is eliminated in BDS-3. Therefore, in Table 4, optimal EWL/WL combinations in different triple-frequency configurations are selected in BDS-3 and Galileo, respectively. The corresponding ambiguity precisions of EWL/WL and the theoretical DD ranges are given. As a comparison, the optimal triple-frequency configuration of GPS is added.

Table 4. Precision of parameters in BDS-3 and Galileo triple-frequency DD configurations.

\begin{tabular}{cccccc}
\hline No. & System & Frequencies & EWL/Cycle & WL/Cycle & DD Range/m \\
\hline 1 & BDS-3 & B1C,B1I,B3I & 0.041 & 0.243 & 0.864 \\
& BDS-3 & B1C,B1I,B2a & 0.041 & 0.314 & 0.939 \\
& BDS-3 & B1C,B3I,B2a & 0.110 & 0.236 & 0.335 \\
& BDS-3 & B1I,B3I,B2a & 0.110 & 0.220 & 0.349 \\
& Galileo & E1,E5a,E5b & 0.043 & 0.358 & 0.635 \\
& Galileo & E1,E5a,E6 & 0.099 & 0.310 & 0.316 \\
& Galileo & E1,E5b,E6 & 0.070 & 0.300 & 0.425 \\
& GPS & L1,L2,L5 & 0.066 & 0.359 & 0.483 \\
\hline
\end{tabular}

It can be seen that the DD ranges of two configurations of triple-frequency signals (B1C,B3I,B2a) and (B1I,B3I,B2a) in BDS-3 are $0.335 \mathrm{~m}$ and $0.349 \mathrm{~m}$, respectively, of which the values are close to the quad-frequency case; therefore, these two configurations of triple-frequency signals can be selected as the optimal triple-frequency signals of BDS-3. As the long baseline is additionally affected by unmodeled errors, when the DD ranges of the two triple-frequency configurations are similar, the configuration with a smaller ambiguity STD is selected. It is easy to determine that the best configuration for Galileo is (E1,E5a,E6) because the ambiguity STD of this configuration is the same as that of quad-frequency, of which the value is $0.316 \mathrm{~m}$. Following Equation (13), the corresponding EWL/WL equation is expressed as follows:

$$
\left[\begin{array}{c}
\boldsymbol{v}_{P}^{C} \\
\boldsymbol{v}_{E W L}^{C} \\
\boldsymbol{v}_{W L}^{C} \\
\boldsymbol{v}_{P}^{E} \\
\boldsymbol{v}_{E W L}^{E} \\
\boldsymbol{v}_{W L}^{E}
\end{array}\right]=\left[\begin{array}{ccccc}
\boldsymbol{e}_{4} \otimes \boldsymbol{B}^{C} & \boldsymbol{\eta}_{P}^{C} \otimes \boldsymbol{I}_{C} & \mathbf{0} & \mathbf{0} & \mathbf{0} \\
\boldsymbol{B}^{C} & -\eta_{E W L} \boldsymbol{I}_{C} & \mathbf{0} & \mathbf{0} & \mathbf{0} \\
\boldsymbol{B}^{C} & -\eta_{W L} \boldsymbol{I}_{C} & \lambda_{W L}^{C} \boldsymbol{I}_{C} & \mathbf{0} & \mathbf{0} \\
\boldsymbol{e}_{4} \otimes \boldsymbol{B}^{E} & \mathbf{0} & \mathbf{0} & \boldsymbol{\eta}_{P}^{E} \otimes \boldsymbol{I}_{e} & \mathbf{0} \\
\boldsymbol{B}^{E} & \mathbf{0} & \mathbf{0} & -\eta_{E W L} \boldsymbol{I}_{e} & \mathbf{0} \\
\boldsymbol{B}^{E} & \mathbf{0} & \mathbf{0} & -\eta_{W L} \boldsymbol{I}_{e} & \lambda_{W L}^{E} \boldsymbol{I}_{e}
\end{array}\right]\left[\begin{array}{c}
\boldsymbol{x} \\
\boldsymbol{i o n}^{C} \\
\boldsymbol{N}_{W}^{C} \\
\boldsymbol{i o n}^{E} \\
\boldsymbol{N}_{W L}^{E}
\end{array}\right]-\left[\begin{array}{c}
\boldsymbol{l}_{P}^{C} \\
\boldsymbol{l}_{E W L}^{C} \\
\boldsymbol{l}_{W L}^{C} \\
\boldsymbol{l}_{P}^{E} \\
\boldsymbol{l}_{E W L}^{E} \\
\boldsymbol{l}_{W L}^{E}
\end{array}\right]
$$

The quad-frequency combination scheme and the optimal triple-frequency combination scheme were selected, corresponding to the No. 1 and No. 3 combination schemes in Table 5. In addition, in Table 4, BDS-3 and Galileo have configurations of triple-frequency signals (B1C,B1I,B2a) and (E1,E5a,E5b) with the worst precision, respectively. It should be noted that 'worst' here refers to the worst of all the combination schemes that have been selected in this experiment, but it is the best in the corresponding triple-frequency combined signals. Coincidentally, they correspond to the quad-frequency combination scheme without the constraints of the fixed second EWL in the second step and directly use fixed EWL to constrain WL ambiguities. Therefore, we added this combination scheme to the comparison as No. 2. Finally, the DD range a priori precisions of the combination of BDS-3 (B1C,B1I,B3I) and the combination of Galileo (E1,E5b,E6) are medium, and they also 
form a combination scheme for comparison, as No. 4. The corresponding coefficients of proposed combination schemes are shown in Table 5.

Table 5. Coefficients of the proposed combination schemes in BDS-3 and Galileo.

\begin{tabular}{|c|c|c|c|c|c|c|c|c|c|c|c|}
\hline \multirow{3}{*}{ No. } & \multirow{3}{*}{$\begin{array}{l}\text { Combination } \\
\text { Schemes }\end{array}$} & \multirow{3}{*}{\multicolumn{2}{|c|}{$\begin{array}{l}\text { Wavelength/m } \\
\text { BDS-3 (Left) } \\
\text { Galileo (Right) }\end{array}$}} & \multicolumn{8}{|c|}{ Satellite Systems } \\
\hline & & & & \multicolumn{4}{|c|}{ BDS-3 } & \multicolumn{4}{|c|}{ Galileo } \\
\hline & & & & $i_{1}$ & $j_{1}$ & $k_{1}$ & $m_{1}$ & $i_{2}$ & $j_{2}$ & $k_{2}$ & $m_{2}$ \\
\hline \multirow[t]{3}{*}{1} & EWL & 20.93 & 9.77 & 1 & -1 & 0 & 0 & 0 & -1 & 1 & 0 \\
\hline & Second EWL & 3.26 & 4.19 & 0 & 0 & 1 & -1 & 0 & 0 & -1 & 1 \\
\hline & WL & 0.78 & 0.75 & 0 & 1 & 0 & -1 & 1 & -1 & 0 & 0 \\
\hline \multirow[t]{2}{*}{2} & EWL & 20.93 & 9.77 & 1 & -1 & 0 & 0 & 0 & -1 & 1 & 0 \\
\hline & WL & 0.78 & 0.75 & 0 & 1 & 0 & -1 & 1 & -1 & 0 & 0 \\
\hline \multirow[t]{2}{*}{3} & EWL & 3.26 & 2.93 & 0 & 0 & 1 & -1 & 0 & -1 & 0 & 1 \\
\hline & $\mathrm{WL}$ & 0.78 & 0.75 & 0 & 1 & 0 & -1 & 1 & -1 & 0 & 0 \\
\hline \multirow[t]{2}{*}{4} & EWL & 20.93 & 4.19 & 1 & -1 & 0 & 0 & 0 & 0 & -1 & 1 \\
\hline & WL & 0.98 & 0.81 & 1 & 0 & -1 & 0 & 1 & 0 & -1 & 0 \\
\hline
\end{tabular}

According to Table 5, there are two EWL combinations in BDS-3; the corresponding wavelengths are, respectively, $20.93 \mathrm{~m}$ and $3.26 \mathrm{~m}$, which are quite different. There are three EWL combinations in Galileo, corresponding to wavelengths of $9.77 \mathrm{~m}, 4.19 \mathrm{~m}$, and $2.93 \mathrm{~m}$. All coefficients are plus or minus 1 or 0 .

\section{Results}

\subsection{Data Description and Processing Strategy}

The BDS-3 and Galileo quad-frequency data were collected on a $104 \mathrm{~km}$ baseline and a $126 \mathrm{~km}$ baseline from 0:00 to 24:00 UTC, the day of year (DOY) 130, 2020, using a pair of Trimble receivers in $\mathrm{Xi}^{\prime}$ an, China, with a sampling interval of $10 \mathrm{~s}$. A total of 8640 epochs were collected, and 26 BDS-3 and 22 Galileo satellites were tracked. The number of BDS-3 and Galileo satellites, with RDOP values as a function of epochs, is shown in Figure 1.



(a)

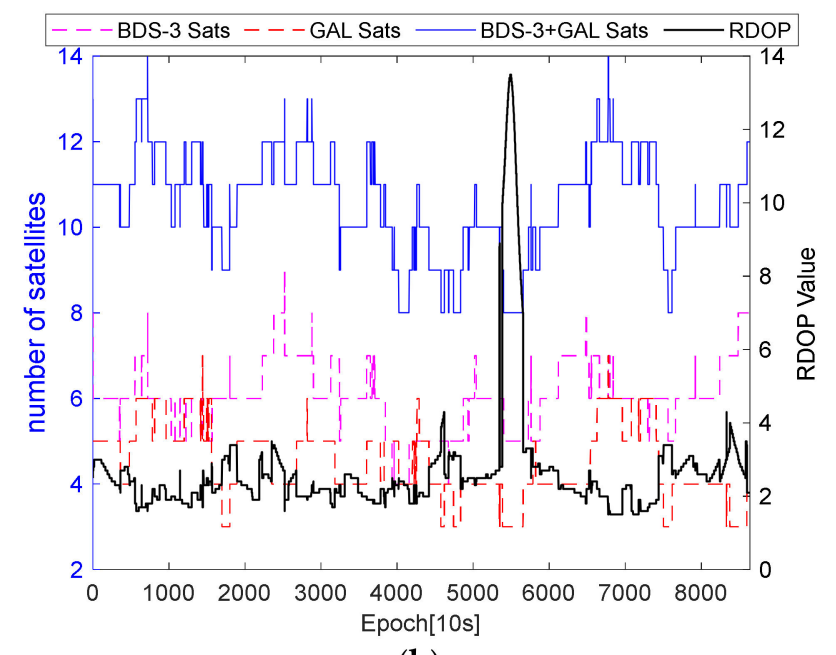

(b)

Figure 1. The number of BDS-3 and Galileo satellites and RDOP values as function of epochs: (a) experiment 1; (b) experiment 2 .

It can be seen from Figure 1a that the number of tracked BDS-3 and Galileo satellites ranged from 9 to 15 and was 12.7 on average during experiment 1 . The minimum number of tracked satellites is 9 around the 4750th epoch and the 5600th epoch, during which RDOP values are greater than 3 . However, the reliability and stability of the long-baseline posi- 
tioning performance is not affected because of a sufficient number of satellites. During the entire observation span, the average RODP value is about 1.85. In experiment 2 , the number of BDS-3 and Galileo satellites is less than that in experiment 1, and the corresponding RDOP value is larger. The average value of the observable RDOP is about 2.61.

The specific processing strategies for long-baseline data are shown in Table 6:

Table 6. Long-baseline data process strategy settings.

\begin{tabular}{cc}
\hline Items & Models \\
\hline Satellites & BDS-3 and Galileo \\
Observations & Carrier phase and observations \\
Frequency selection & BDS-3: B1C/B1I/B3I/B2a; Galileo: \\
Cutoff elevation & E1/E5a/E5b/E6 \\
Sampling rate & $10^{\circ}$ \\
& $10 \mathrm{~s}$ \\
Observation weighting & Elevation-dependent weighting \\
& Relative weighting of code and phase \\
Satellite orbit & observations: $1: 100$ \\
Satellite clock & Broadcast ephemeris \\
Receiver clock & DD eliminated or weakening \\
Ionospheric delay & DD eliminated or weakening \\
Tropospheric delay & Estimated as parameters \\
Phase ambiguities & Corrected by GPT2 $w$ model $[30]$ \\
\hline
\end{tabular}

\subsection{Performance of EWL AR for BDS-3 and Galileo}

We analyzed the data of the entire observation span and plotted the single-epoch ambiguity decimal fraction of the "true" ambiguities and float ambiguities of the EWL of BDS-3 and Galileo satellite pairs. According to Table 5, the EWL signals of combination schemes No. 1, No. 2, and No. 4 are the same in BDS-3, of which the wavelength is $20.93 \mathrm{~m}$. The EWL signal wavelength of combination scheme No. 3 is $3.26 \mathrm{~m}$. In Galileo, the EWL signals of combination schemes No. 1 and No. 2 are the same, of which the wavelength is $9.77 \mathrm{~m}$. The EWL signal wavelength of combination scheme No. 3 is $2.93 \mathrm{~m}$, and that of combination scheme No. 4 is $4.19 \mathrm{~m}$. The EWL ambiguity fractions of the BDS-3 and Galileo satellite pairs for all epochs are shown in Figures 2 and 3, and different colors represent different satellite pairs.

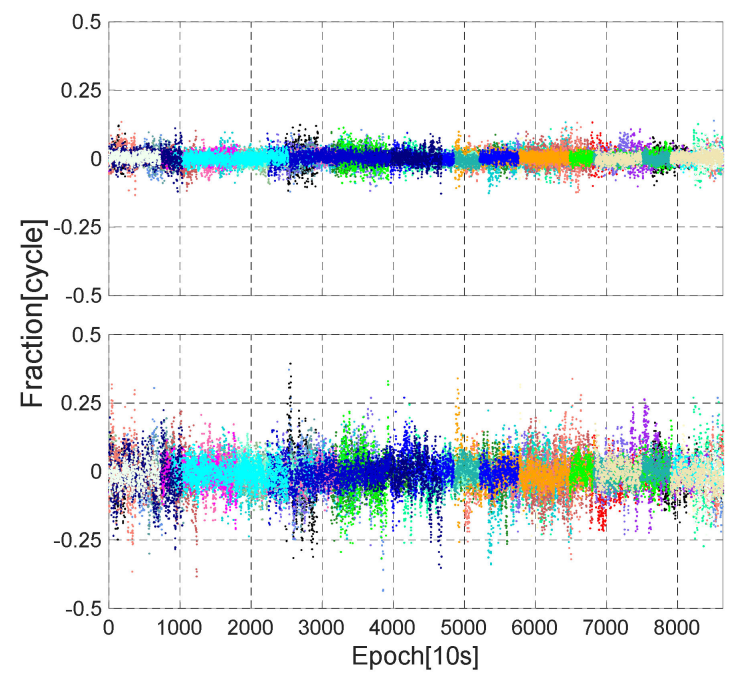

(a)

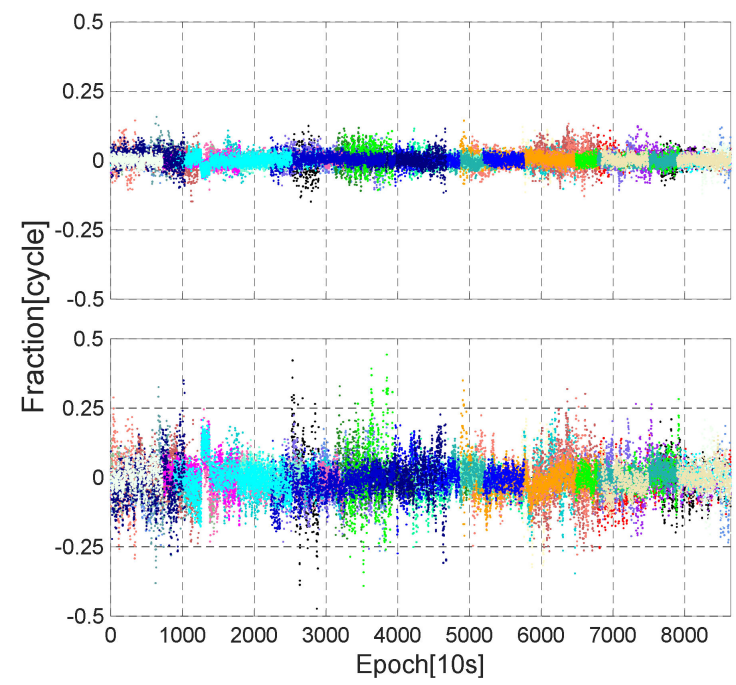

(b)

Figure 2. EWL ambiguity fractions of combination schemes No. 1, No. 2, No. 4 (top), and No. 3 (bottom) for BDS-3: (a) experiment 1; (b) experiment 2. Each color denotes one satellite pair. 

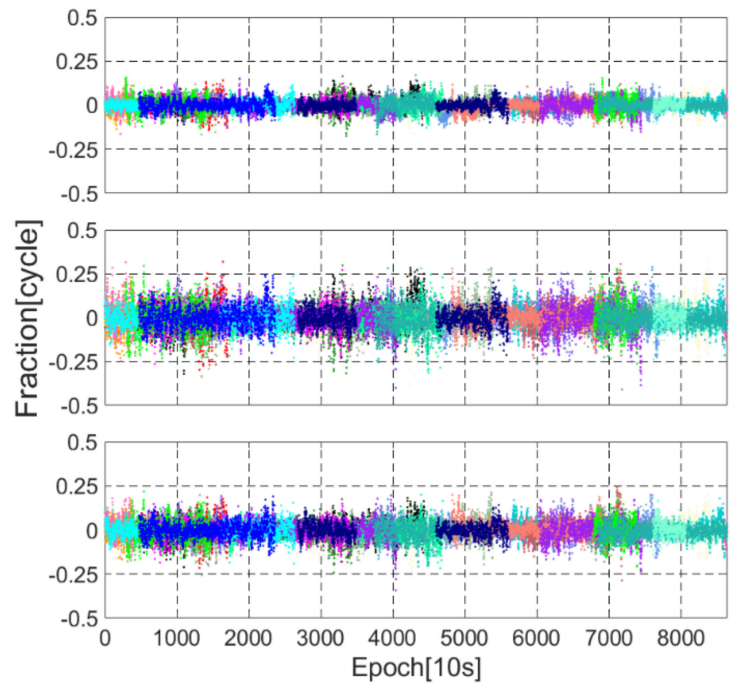

(a)


(b)

Figure 3. EWL ambiguity fractions of combination schemes No. 1, No. 2 (top), No. 3 (middle), and No. 4 (bottom) for Galileo: (a) experiment 1; (b) experiment 2. Each color denotes one satellite pair.

It can be seen that the ambiguity fractions of EWL have a strong correlation with their wavelengths: the longer the wavelength, the smaller ambiguity fractions. The ambiguity fractions of EWL between BDS-3 and Galileo satellite pairs are mostly less than 0.25 cycles; especially BDS-3 $(1,-1,0,0)$ and Galileo $(0,-1,1,0)$ are less than 0.1 cycles, which are consistent with the description of Tables 2 and 3 in Section 2.4. Table 7 counts the mean and STD values of the EWL ambiguity fractions. It can be found that the mean and STD values of the five EWL ambiguity fractions are less than 0.01 and 0.07 cycles, of which $\operatorname{BDS}-3(1,-1,0,0)$ is only 0.002 and 0.021 cycles and Galileo $(0,-1,0,1)$ is -0.001 and 0.030 cycles, respectively. This indicates that the EWL ambiguities of BDS-3 and Galileo can be fixed instantaneously. Therefore, the application of EWL AR for large scales and complex environments has great potential. Compared with Tables 2-4, it can be found that the EWL ambiguity fraction STD values of the experimental results are smaller than the theoretical values. The reason for this is that the actual pseudo-range and carrier phase precision are higher than our preset [31-33].

Table 7. Mean and STD values for EWL ambiguity fractions of BDS-3 and Galileo in experiment 1 and experiment 2 .

\begin{tabular}{clccccc}
\hline \multirow{2}{*}{ Experiment } & \multirow{2}{*}{ Statistics } & \multicolumn{2}{c}{ BDS-3 } & \multicolumn{3}{c}{ Galileo } \\
\cline { 3 - 7 } & & $\mathbf{( 1 , - 1 , 0 , 0 )}$ & $\mathbf{( 0 , 0 , 1 , - 1 )}$ & $\mathbf{( 0 , - 1 , 1 , 0 )}$ & $\mathbf{( 0 , - 1 , 0 , 1 )}$ & $\mathbf{( 0 , 0 , - , 1 )}$ \\
\hline \multirow{2}{*}{1} & Mean/cycle & 0.002 & -0.010 & -0.001 & -0.002 & -0.002 \\
& STD/cycle & 0.021 & 0.058 & 0.030 & 0.063 & 0.046 \\
2 & Mean/cycle & 0.004 & -0.013 & 0.001 & 0.001 & -0.001 \\
& STD/cycle & 0.023 & 0.064 & 0.029 & 0.063 & 0.046 \\
\hline
\end{tabular}

\subsection{Performance of WL AR for BDS-3 and Galileo}

The WL ambiguity fractions of the two experiments and corresponding fraction histograms of BDS-3 and Galileo satellite pairs are shown in Figures 4-7. Figures 4 and 5 show the WL ambiguity fractions of BDS-3 and Galileo corresponding to experiment 1, respectively. It can be clearly seen that the fractional parts of the WL ambiguities corresponding to the four combinations of BDS-3 and Galileo are almost all distributed with zero mean. In particular, the ambiguity fractions of combination schemes No. 1 and No. 3 (corresponding to the first one and the third one in Figures 4 and 5, respectively) are mostly less than 0.3 cycles, which shows the feasibility of a single-epoch WL AR. The ambiguity fractions 
of combination scheme No. 2 are obviously more scattered than the other three schemes. Figures 6 and 7 show the WL ambiguity fractions of BDS-3 and Galileo correspond to experiment 2 , respectively. It can be seen that the WL ambiguity fractions of experiment 2 are more scattered compared to that of experiment 1 and have a great relationship with the worst observation data.

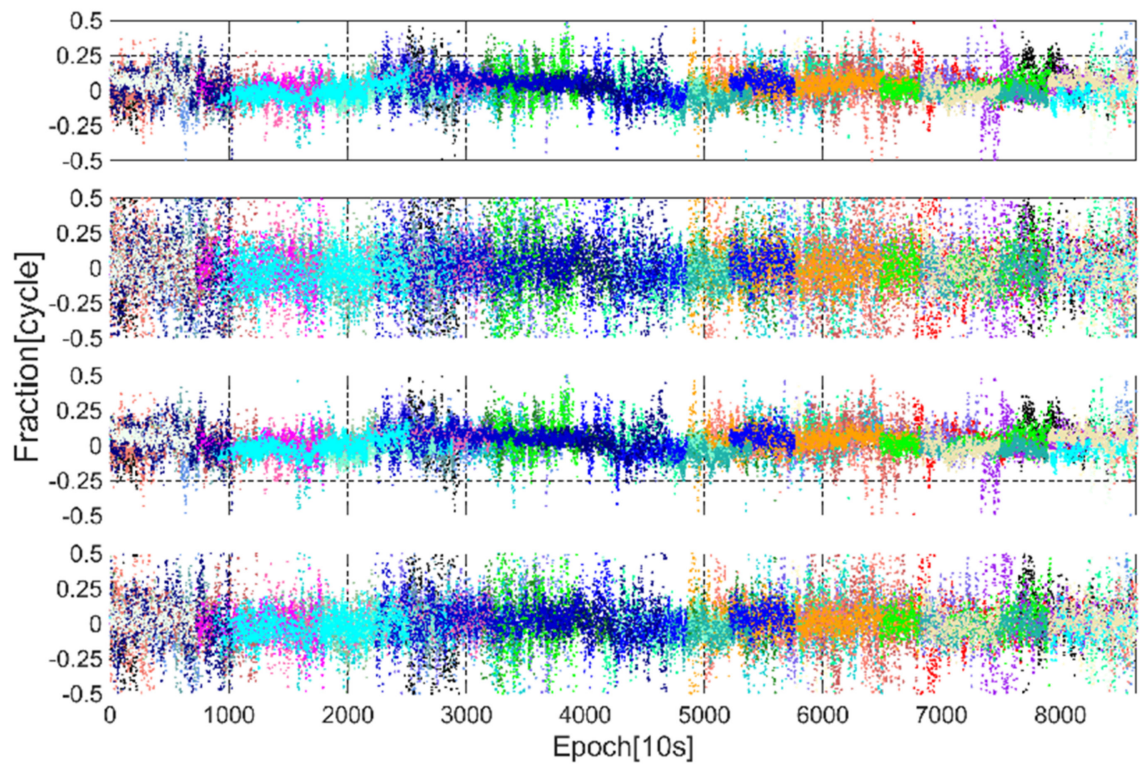

(a)

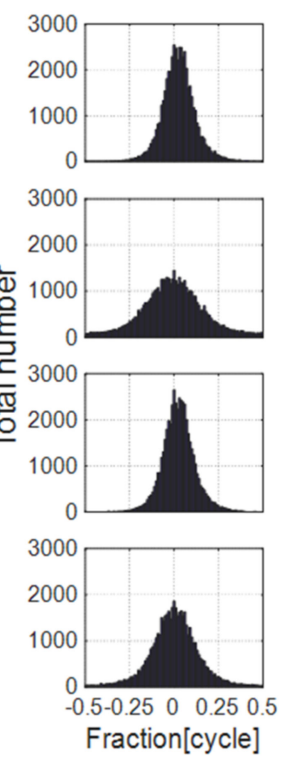

(b)

Figure 4. Experiment 1: WL ambiguity fractions and the corresponding histograms of combination schemes No. 1, No. 2, No. 3, and No. 4 (from top to bottom) for BDS-3: (a) WL ambiguity fractions, each color denotes one satellite pair; (b) WL ambiguity fraction statistics histograms.

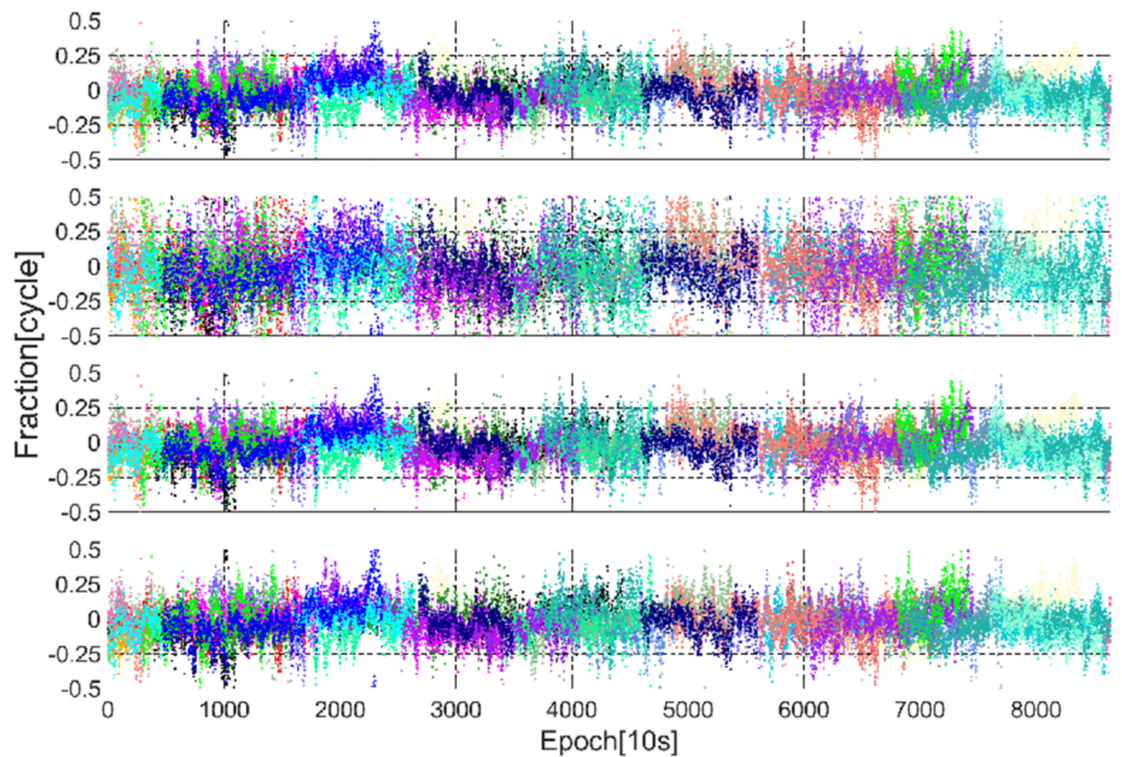

(a)

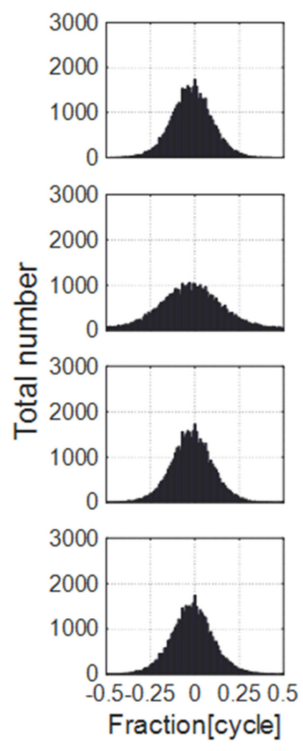

(b)

Figure 5. Experiment 1: WL ambiguity fractions and the corresponding histograms of combination schemes No. 1, No. 2, No. 3, and No. 4 (from top to bottom) for Galileo: (a) WL ambiguity fractions, each color denotes one satellite pair; (b) WL ambiguity fraction statistics histograms. 




(a)



(b)

Figure 6. Experiment 2: WL ambiguity fractions and the corresponding histograms of combination schemes No. 1, No. 2, No. 3, and No. 4 (from top to bottom) for Galileo: (a) WL ambiguity fractions, each color denotes one satellite pair; (b) WL ambiguity fraction statistics histograms.

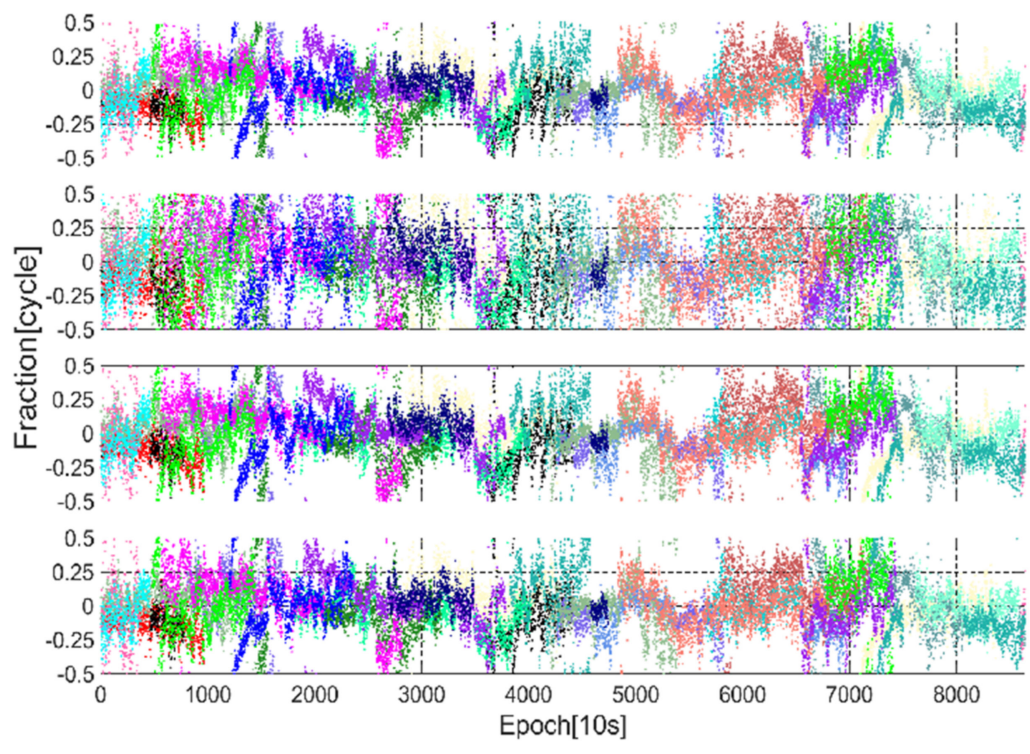

(a)

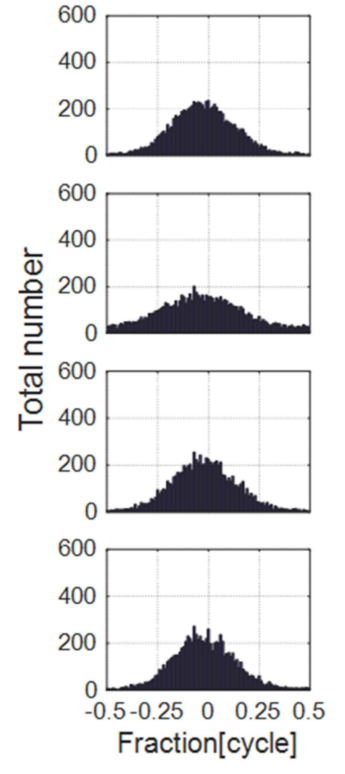

(b)

Figure 7. Experiment 2: WL ambiguity fractions and the corresponding histograms of combination schemes No. 1, No. 2, No. 3, and No. 4 (from top to bottom) for Galileo: (a) WL ambiguity fractions, each color denotes one satellite pair; (b) WL ambiguity fraction statistics histograms.

Table 8 shows the mean and STD values of the WL ambiguity fractions. It can be found that, in experiment 1 , the mean and STD values of the five WL ambiguity fractions are less than 0.03 and 0.2 cycles, respectively. The ambiguity fraction STD value of combination scheme No. 3 is almost equal to that of combination scheme No. 1. In general, the above discussion is still applied in experiment 2, although the STD values of experiment 2 are larger than that of experiment 1 . 
Table 8. Mean and STD values for WL ambiguity fractions of BDS-3 and Galileo in experiment 1 and experiment 2.

\begin{tabular}{cccccccccc}
\hline \multirow{2}{*}{ Experiment } & Combination & \multicolumn{4}{c}{ BDS-3 } \\
\cline { 3 - 9 } & Scheme (No.) & No. 1 & No. 2 & No. 3 & No. 4 & No. 1 & No. 2 & No. 3 & No. 4 \\
\hline \multirow{2}{*}{1} & Mean/cycle & 0.026 & -0.006 & 0.025 & 0.002 & -0.018 & -0.020 & -0.018 & -0.016 \\
& STD/cycle & 0.094 & 0.175 & 0.095 & 0.139 & 0.115 & 0.182 & 0.117 & 0.121 \\
2 & Mean/cycle & 0.046 & -0.005 & 0.046 & 0.011 & -0.024 & -0.030 & -0.020 & -0.020 \\
& STD/cycle & 0.123 & 0.176 & 0.123 & 0.147 & 0.160 & 0.215 & 0.163 & 0.158 \\
\hline
\end{tabular}

3.4. Results and Analyses of Long-Baseline RTK Positioning

Figure 8 shows the results of experiment 1 calculated by combination schemes No. 1 , No. 2, No. 3, and No. 4. The corresponding root mean square (RMS) errors of three directions-east $(\mathrm{E})$, north $(\mathrm{N})$, and up (U) —and in 3D are shown in Table 9.

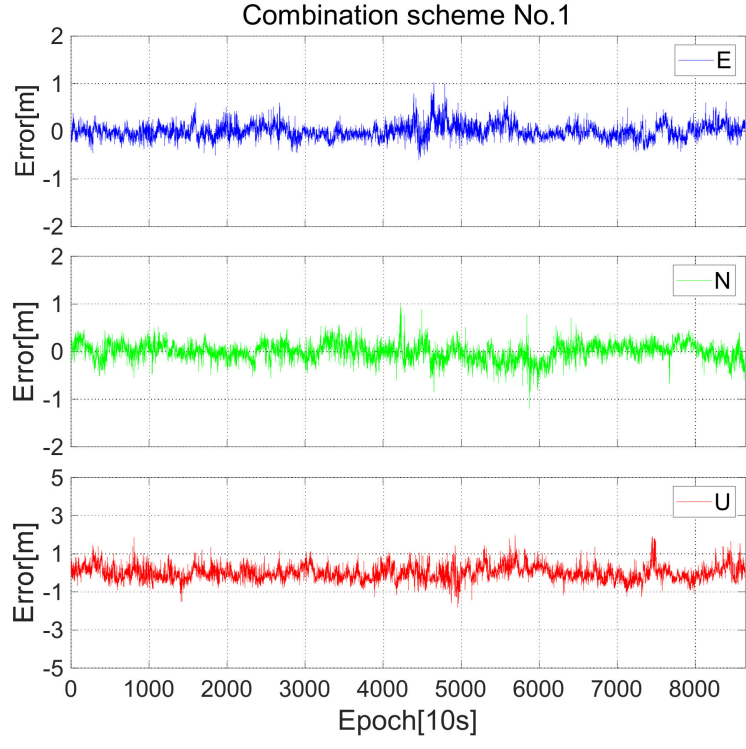

(a)

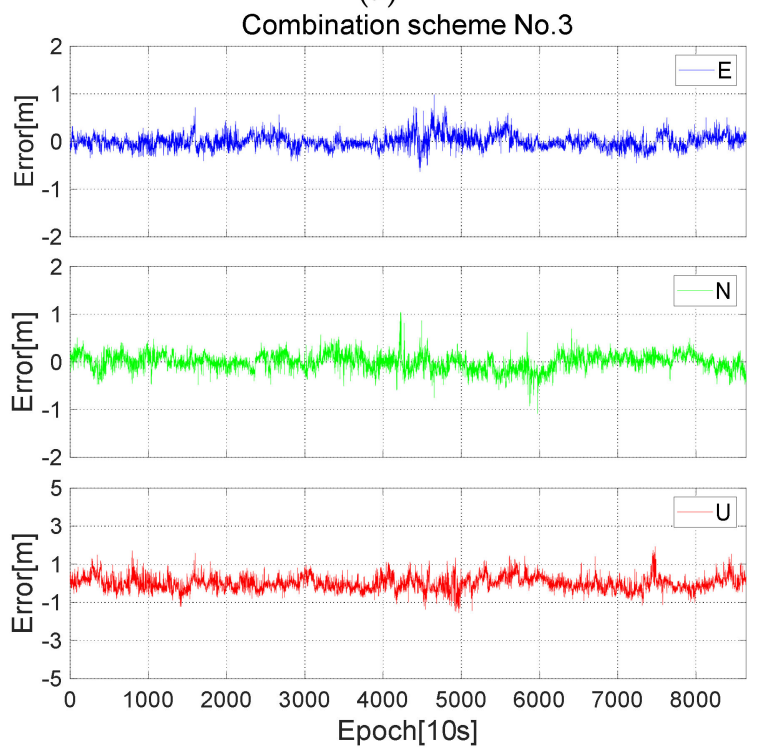

(c)

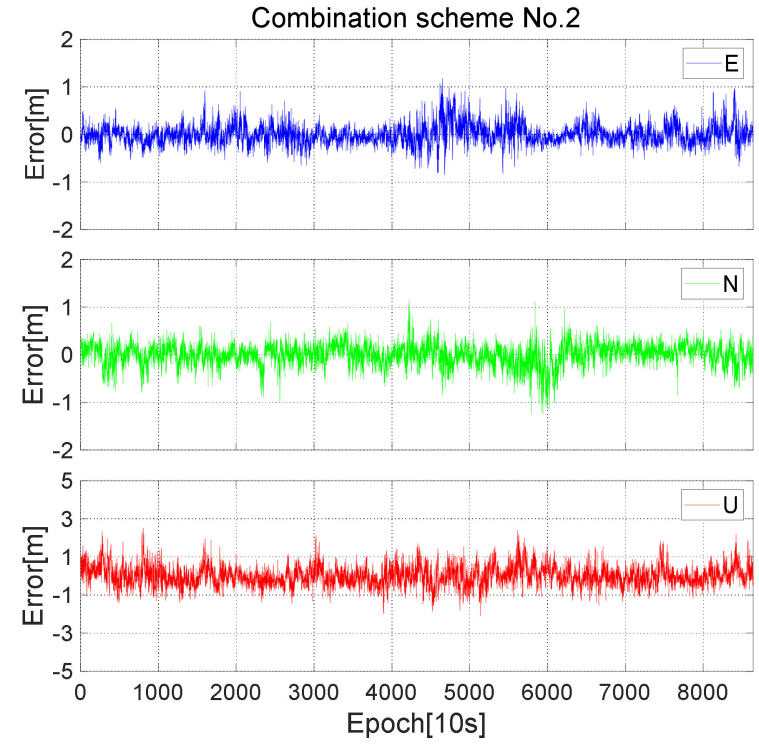

(b)

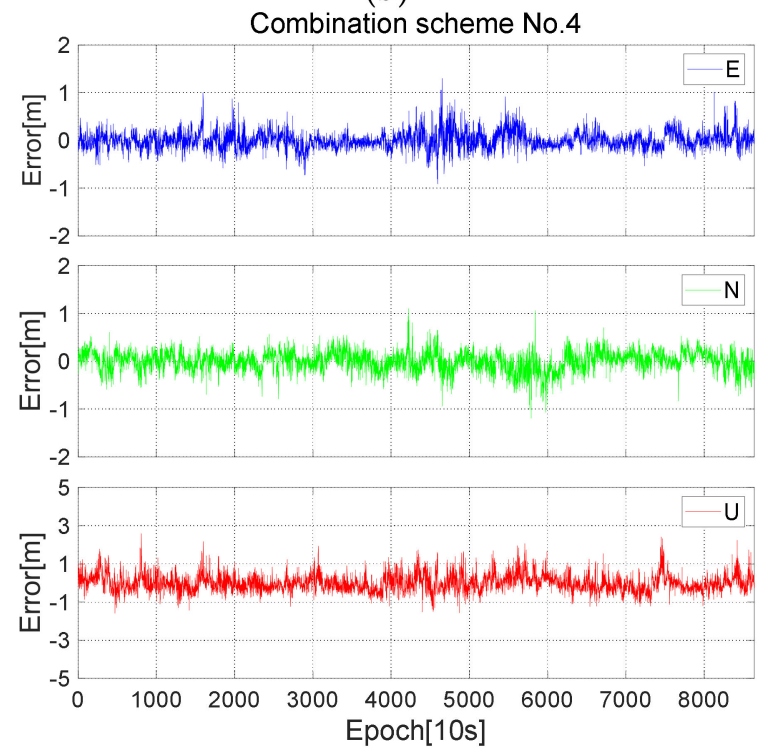

(d)

Figure 8. Experiment 1: long-baseline positioning performance of four combination schemes of BDS-3 + Galileo: (a) combination scheme No. 1, (b) combination scheme No. 2, (c) combination scheme No. 3, and (d) combination scheme No. 4. 
Table 9. The statistical RMS results of the positioning differences in experiment 1.

\begin{tabular}{ccccc}
\hline \multirow{2}{*}{$\begin{array}{c}\text { Combination } \\
\text { Scheme (No.) }\end{array}$} & E & N & U & 3D \\
\cline { 2 - 5 } & 14.5 & 17.2 & 37.4 & 43.6 \\
1 & 20.8 & 23.1 & 52.9 & 61.4 \\
2 & 14.5 & 17.2 & 37.3 & 43.6 \\
3 & 17.9 & 20.3 & 44.5 & 52.1 \\
\hline
\end{tabular}

It can be seen that the positioning precision of combination schemes No. 1 and No. 3 are almost the same, with a $22.5 \mathrm{~cm}$ plane precision. The positioning precision of combination scheme No. 4 is in the second place, with a $27.1 \mathrm{~cm}$ plane precision, and the precision of combination scheme No. 2 is the worst, which is about $31.1 \mathrm{~cm}$. Table 10 shows the percentage of past ratio-test values with the given thresholds. Combination scheme No. 3 still has a $99 \%$ passing rate under the ratio-test value of 2.5 , even better than the performance of combination scheme No. 1. However, combination schemes No. 2 and No. 4 are both lower than $90 \%$ under the same ratio-test value.

Table 10. The percentage of past ratio-test values with the given thresholds in experiment 1 .

\begin{tabular}{ccccc}
\hline $\begin{array}{c}\text { Combination } \\
\text { Scheme (No.) }\end{array}$ & $\boldsymbol{R}>\mathbf{1 . 5 ( \% )}$ & $\boldsymbol{R}>\mathbf{2}(\mathbf{\%})$ & $\boldsymbol{R}>\mathbf{2 . 5 ( \% )}$ & $\boldsymbol{R}>\mathbf{3}(\mathbf{\%})$ \\
\hline 1 & 100.0 & 99.9 & 98.9 & 96.0 \\
2 & 99.5 & 95.5 & 88.1 & 80.0 \\
3 & 100.0 & 99.9 & 99.0 & 96.2 \\
4 & 99.4 & 93.1 & 83.4 & 73.6 \\
\hline
\end{tabular}

Figure 9 shows the results of experiment 2 calculated using combination schemes No. 1, No. 2, No. 3, and No. 4. The corresponding RMS errors of the three directions E, N, and $\mathrm{U}$ and in 3D are shown in Table 11.

It can be seen that the positioning deviation of Experiment 2 is significantly larger than that of experiment 1 , which is consistent with the previous discussion. Similar to that in experiment 1, the positioning precisions of combination schemes No. 1 and No. 3 are almost the same as that in experiment 2, and the plane precision is about $43.2 \mathrm{~cm}$. The positioning precision of combination scheme No. 4 is in second place, with a $50.4 \mathrm{~cm}$ plane precision. The precision of combination scheme No. 2 is the worst and is about $51.9 \mathrm{~cm}$. Table 12 shows the percentage of past ratio-test values with the given thresholds. Combination scheme No. 3 still has a 99\% passing rate under the ratio-test value of 2 and is close to that of combination scheme No. 1 under all given thresholds.

Table 11. The statistical RMS results of the positioning differences in experiment 2.

\begin{tabular}{ccccc}
\hline \multirow{2}{*}{$\begin{array}{c}\text { Combination } \\
\text { Scheme (No.) }\end{array}$} & $\mathbf{5}$ & $\mathbf{N}$ & $\mathbf{U}$ & RMS (cm) \\
\cline { 2 - 5 } & 27.7 & 32.6 & 68.4 & 80.7 \\
1 & 35.5 & 37.8 & 86.3 & 100.7 \\
2 & 28.0 & 32.9 & 69.7 & 81.9 \\
3 & 33.5 & 37.6 & 80.6 & 95.0 \\
\hline
\end{tabular}




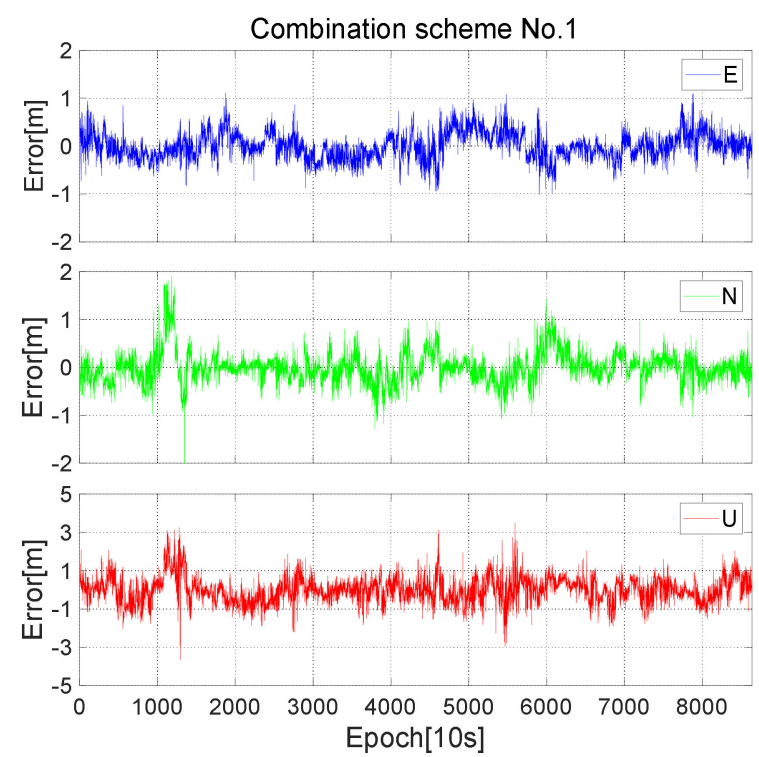

(a)

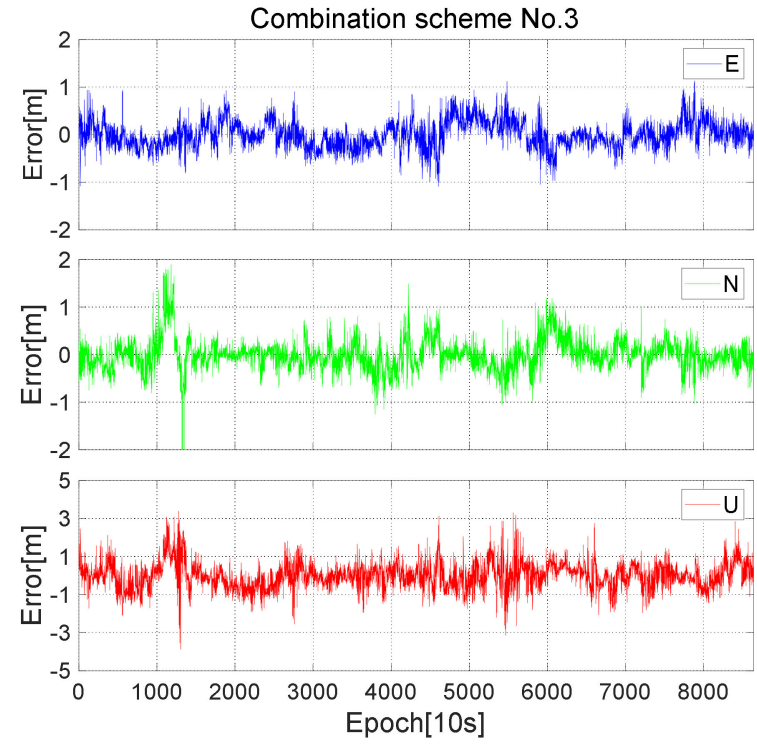

(c)

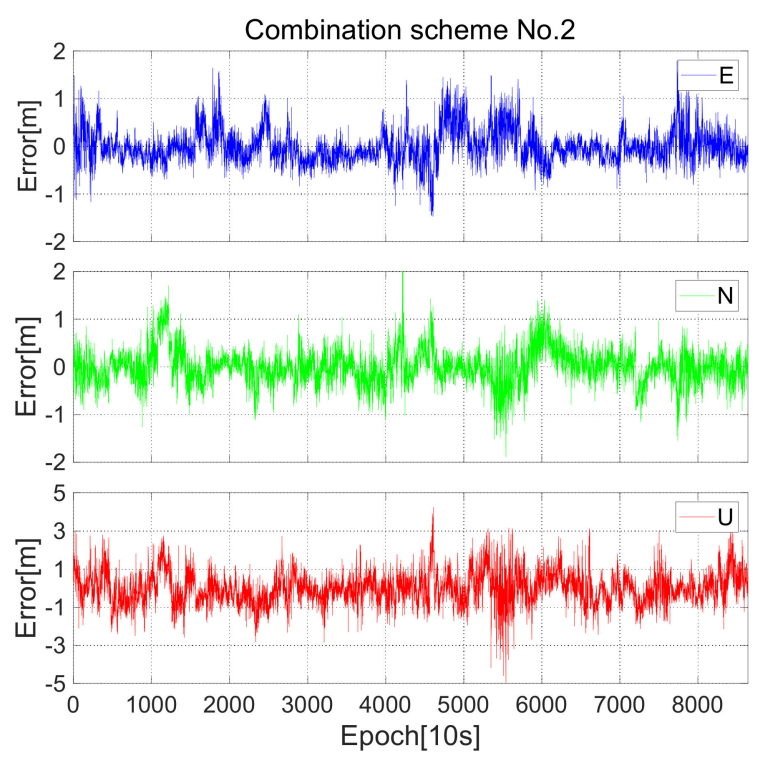

(b)

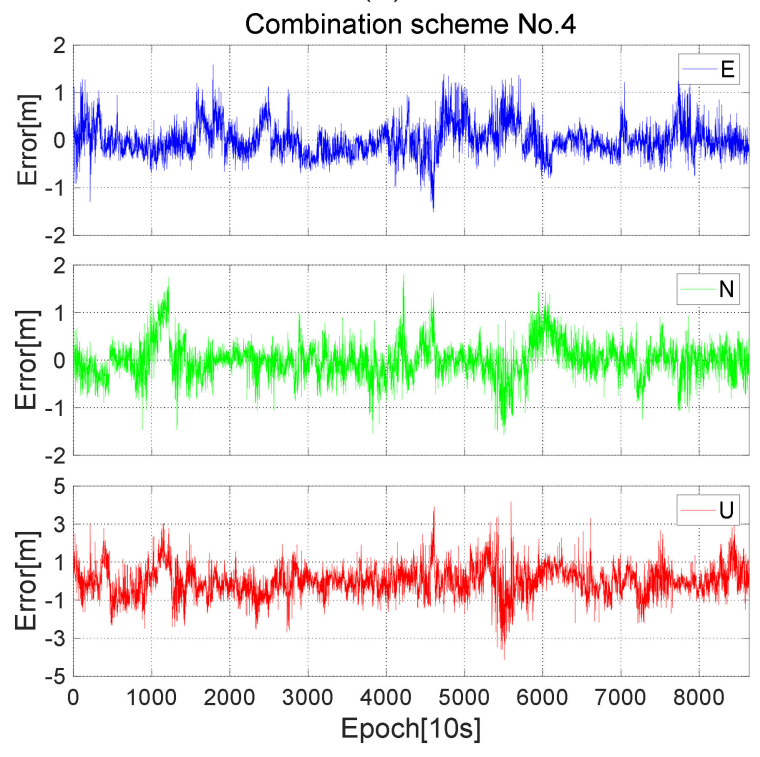

(d)

Figure 9. Experiment 2: long-baseline positioning performance of four combination schemes of BDS-3 + Galileo: (a) combination scheme No. 1, (b) combination scheme No. 2, (c) combination scheme No. 3, and (d) combination scheme No. 4.

Table 12. The percentage of past ratio-test values with given thresholds in experiment 2.

\begin{tabular}{ccccc}
\hline $\begin{array}{c}\text { Combination } \\
\text { Scheme (No.) }\end{array}$ & $\boldsymbol{R}>\mathbf{1 . 5 ( \% )}$ & $\boldsymbol{R}>\mathbf{2}(\mathbf{\%})$ & $\boldsymbol{R}>\mathbf{2 . 5 ( \% )}$ & $\boldsymbol{R}>\mathbf{3}(\mathbf{\%})$ \\
\hline 1 & 100.0 & 99.2 & 96.1 & 91.9 \\
2 & 97.8 & 95.7 & 91.2 & 86.2 \\
3 & 99.9 & 99.0 & 95.8 & 91.7 \\
4 & 99.4 & 95.3 & 88.6 & 80.5 \\
\hline
\end{tabular}

\section{Discussion}

Since the long-baseline positioning performance is mainly affected by the measurement noise of observation data, the atmospheric residuals, and the orbital residuals, it is necessary to evaluate the a priori success rate of the EWL combination in Section 3.2 under different error values. The a priori precision of code and carrier depend on the observation 
environments and the types of receivers. Assuming that the receivers used are professional geodetic receivers and that the observation condition is an open field, the DD pseudo-range and carrier precision are usually set as $0.5 \mathrm{~m}$ and $5 \mathrm{~mm}$, respectively [32,34].

As shown in Figure 10, it is the relationship between the success rate of the EWL signals and the total errors in the theoretical situation. The total errors here include the second-order ionospheric residuals, the troposphere residuals, and the orbital residuals. It can be seen that, in theory, BDS-3 $(1,-1,0,0)$ and Galileo $(0,-1,1,0)$ can still maintain success rates of over $99 \%$ when the total errors reach $35 \mathrm{~cm}$. The remaining EWL signals BDS-3 $(0,0,1,-1)$, and Galileo $(0,0,-1,1)$ and $(0,-1,0,1)$ have success rates of more than $99 \%$ when the total errors reach $24 \mathrm{~cm}$, which meet the important condition of long-baseline reliable positioning.

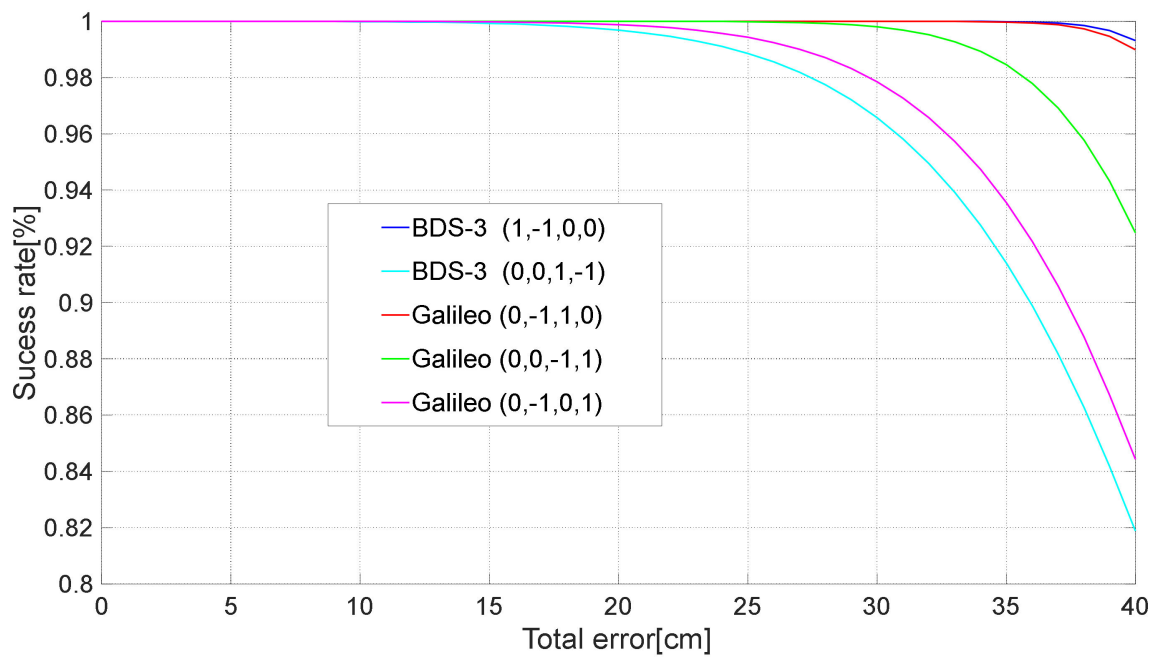

Figure 10. The success rate of theoretical EWL AR under different total errors.

It can be known from Reference [16] that, for a long baseline from $100 \mathrm{~km}$ to $500 \mathrm{~km}$, the total errors range between $4.5 \mathrm{~cm}$ and $32 \mathrm{~cm}$. Therefore, BDS-3 $(1,-1,0,0)$ and Galileo $(0,-1,1,0)$ can reliably fix the long-baseline instantaneously. Generally, the troposphere residuals of the long baseline do not reach the maximum value of $20 \mathrm{~cm}$ from Reference [16] but reach the more common $10 \mathrm{~cm}$ [35]. If more precise ephemeris data are used, the total errors are between $13 \mathrm{~cm}$ and $22 \mathrm{~cm}$. The success rates of BDS-3 $(0,0,1,-1)$, and Galileo $(0,0,-1,1)$ and $(0,-1,0,1)$ are greater than $99.4 \%$, which can be fixed instantaneously in the first step. Therefore, the five EWL signals of BDS-3 and Galileo mentioned above can reliably fix the long baseline instantaneously.

Therefore, the proposed optimal triple-frequency EWL/WL combinations with (B1I,B3I, B2a) and (E1,E5a,E6) signals are suitable for most long baselines, which can obtain the same precision as using four frequencies.

\section{Conclusions}

In this study, founded on the principles of a small noise amplitude factor and a small ionospheric scalar factor, we selected the optimal triple-frequency EWL/WL signals (B1I,B3I,B2a) of BDS-3 and (E1,E5a,E6) of Galileo in the four frequencies. A long-baseline AR method based on optimal triple-frequency EWL/WL combinations was proposed. In order to test the positioning performance and AR of the optimal triple-frequency EWL/WL combinations, two configurations of typical triple-frequency EWL/WL signals and quadfrequency EWL/Second EWL/WL signals were added.

From all the discussion above, we can draw the following conclusions:

1. From the experimental results of the $104 \mathrm{~km}$ and $126 \mathrm{~km}$ baselines, it was shown that the ambiguities of EWL signals mentioned in this paper can be fixed reliably in single-epoch, whether it is BDS-3 or Galileo. 
2. The optimal triple-frequency EWL/WL combinations with (B1I,B3I,B2a) and (E1,E5a,E6) signals can achieve the same precision as that with quad-frequency signals, which can be applied to most long-baselines with a success rate of more than $99.4 \%$.

3. This method can achieve instantaneous precision at the decimeter level and can be applied to large-range network RTK positioning, long-distance navigation, and positioning in open environments. In the application of relative positioning, filtering and smoothing methods can also be used to achieve higher precision positioning results.

4. With the increase in available satellites and satellite frequencies in the future, the dimension of matrix will be greater. It is necessary to eliminate redundant frequencies when calculate the multi-dimensional matrix. Selecting the optimal frequencies can greatly reduce the dimension of matrix and is an effective method.

Author Contributions: L.L. conceived the idea and designed the experiments with S.P. and W.G., L.L. wrote the main manuscript. C.M., J.T., and Q.Z. reviewed the paper. All components of this research were carried out under the supervision of L.L. All authors have read and agreed to the published version of the manuscript.

Funding: This research was funded by the National Natural Science Foundation of China (grant No. 41904022 and 41774027) and by the National Key Technologies R\&D Program (grant No. 2016YFB0502101).

Institutional Review Board Statement: Not applicable.

Informed Consent Statement: Not applicable.

Data Availability Statement: The data presented in this study are available upon request from the corresponding author. The data are not publicly available due to security issues.

Acknowledgments: We thank all the scientists and principal researchers who prepared and provided the research data. We would also like to thank our colleagues from the School of Instrument Science and Engineering who provided profound insights and expertise to our research.

Conflicts of Interest: The authors declare no conflict of interest.

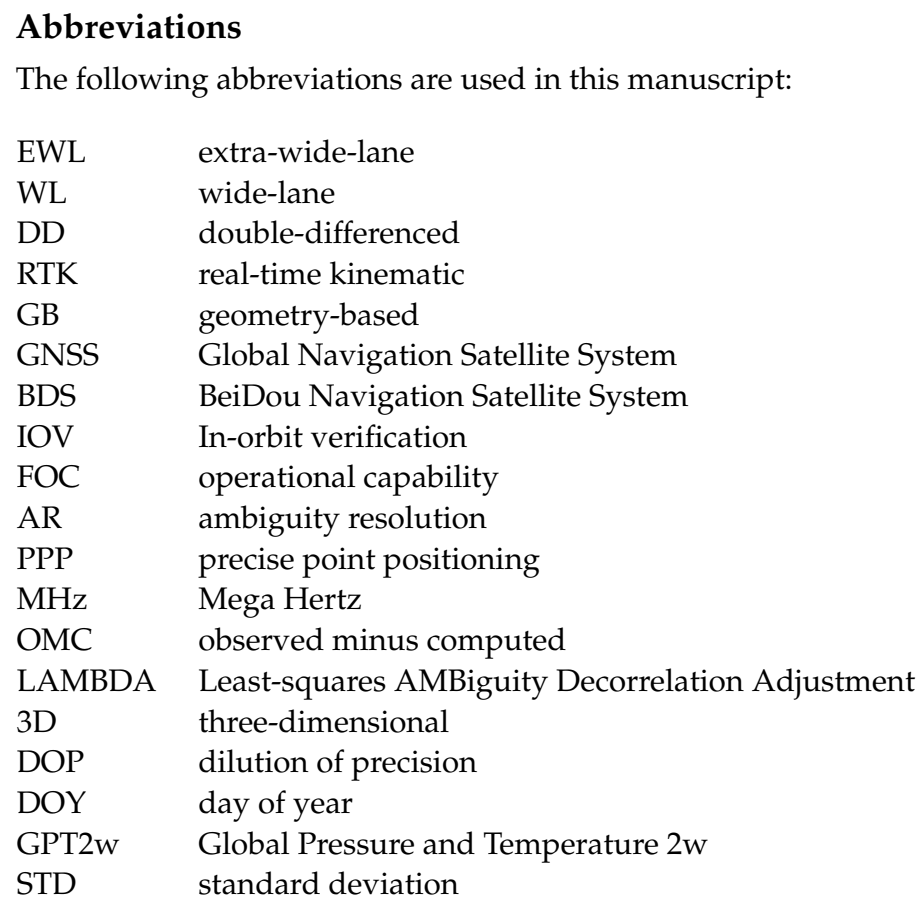




\section{References}

1. Tu, R.; Liu, J.; Zhang, R.; Zhang, P.; Huang, X.; Lu, X. RTK model and positioning performance analysis using Galileo fourfrequency observations. Adv. Space Res. 2019, 63, 913-926. [CrossRef]

2. Li, X.; Liu, G.; Li, X.; Zhou, F.; Feng, G.; Yuan, Y.; Zhang, K. Galileo PPP rapid ambiguity resolution with five-frequency observations. GPS Solut. 2020, 24, 24. [CrossRef]

3. Miao, W.; Li, B.; Zhang, Z.; Zhang, X. Combined BeiDou-2 and BeiDou-3 instantaneous RTK positioning: Stochastic modeling and positioning performance assessment. J. Spat. Sci. 2020, 65, 7-24. [CrossRef]

4. Wang, J.; Huang, G.; Zhang, Q.; Gao, Y.; Gao, Y.; Luo, Y. GPS/BDS-2/Galileo Precise Point Positioning Ambiguity Resolution Based on the Uncombined Model. Remote Sens. 2020, 12, 1853. [CrossRef]

5. Gao, W.; Meng, X.; Gao, C.; Pan, S.; Wang, D. Combined GPS and BDS for single-frequency continuous RTK positioning through real-time estimation of differential inter-system biases. GPS Solut. 2018, 22, 20. [CrossRef]

6. Zhang, Z.; Li, B.; He, X. Geometry-free single-epoch resolution of BDS-3 multi-frequency carrier ambi-guities. Acta Geod. Cartogr. Sin. 2020, 49, 1139-1148. [CrossRef]

7. Wang, K.; Khodabandeh, A.; Teunissen, P.J. Five-frequency Galileo long-baseline ambiguity resolution with multipath mitigation. GPS Solut. 2018, 22, 75. [CrossRef]

8. Hou, P.; Zhang, B.; Yuan, Y. Combined GPS+ BDS instantaneous single-and dual-frequency RTK positioning: Stochastic modelling and performance assessment. J. Spat. Sci. 2019, 1-24. [CrossRef]

9. Pan, L.; Xiaohong, Z.; Fei, G. Ambiguity resolved precise point positioning with GPS and BeiDou. J. Geodesy 2017, 91, 25-40. [CrossRef]

10. Zhao, Q.; Gao, W.; Gao, C.; Pan, S.; Yang, X.; Wang, J. Comprehensive outage compensation of real-time orbit and clock corrections with broadcast ephemeris for ambiguity-fixed precise point positioning. Adv. Space Res. 2020, 67, 1124-1142. [CrossRef]

11. Laurichesse, D.; Banville, S. Innovation: Instantaneous centimeter-level multi-frequency precise point positioning. GPS World J. 2018, 29, 43-47.

12. Geng, J.; Meng, X.; Dodson, A.H.; Ge, M.; Teferle, F.N. Rapid re-convergences to ambiguity-fixed solutions in precise point positioning. J. Geodesy 2010, 84, 705-714. [CrossRef]

13. Geng, J.; Guo, J.; Chang, H.; Li, X. Toward global instantaneous decimeter-level positioning using tightly coupled multiconstellation and multi-frequency GNSS. J. Geodesy 2019, 93, 977-991. [CrossRef]

14. Hatch, R.; Jung, J.; Enge, P.; Pervan, B. Civilian GPS: The benefits of three frequencies. GPS Solut. 2000, 3, 1-9. [CrossRef]

15. Geng, J.; Bock, Y. Triple-frequency GPS precise point positioning with rapid ambiguity resolution. J. Geodesy 2013, 87, 449-460. [CrossRef]

16. Feng, Y. GNSS three carrier ambiguity resolution using ionosphere-reduced virtual signals. J. Geodesy 2008, 82, 847-862. [CrossRef]

17. Montenbruck, O.; Hauschild, A.; Steigenberger, P.; Hugentobler, U.; Teunissen, P.; Nakamura, S. Initial assessment of the COMPASS/BeiDou-2 regional navigation satellite system. GPS Solut. 2013, 17, 211-222. [CrossRef]

18. Li, B.; Feng, Y.; Gao, W.; Li, Z. Real-time kinematic positioning over long baselines using triple-frequency BeiDou signals. IEEE Trans. Aerosp. Electron. Syst. 2015, 51, 3254-3269. [CrossRef]

19. Feng, Y.; Li, B. A benefit of multiple carrier GNSS signals: Regional scale network-based RTK with doubled inter-station distances. J. Spat. Sci. 2008, 53, 135-147. [CrossRef]

20. Gao, W.; Gao, C.; Pan, S.; Wang, D.; Wang, S. Single-epoch positioning method in network RTK with BDS triple-frequency widelane combinations. Acta Geod. Cartogr. Sin. 2015, 44, 641-648. [CrossRef]

21. Li, B.; Zhang, Z.; Miao, W. Improved precise positioning with BDS-3 quad-frequency signals. Satell. Navig. 2020, 1, 1-10. [CrossRef]

22. Li, X.; Li, X.; Liu, G.; Feng, G.; Yuan, Y.; Zhang, K.; Ren, X. Triple-frequency PPP ambiguity resolution with multi-constellation GNSS: BDS and Galileo. J. Geodesy 2019, 93, 1105-1122. [CrossRef]

23. Tang, W.; Deng, C.; Shi, C.; Liu, J. Triple-frequency carrier ambiguity resolution for Beidou navigation satellite system. GPS Solut. 2014, 18, 335-344. [CrossRef]

24. Teunissen, P.J.; De Jonge, P.J.; Tiberius, C.C.J.M. Performance of the LAMBDA method for fast GPS ambiguity resolution. Navigation 1997, 44, 373-383. [CrossRef]

25. Li, Z.; Huang, J. GPS Surveying and Data Processing; Wuhan University Press: Wuhan, China, 2005; pp. $188-189$.

26. Bard, J.D.; Ham, F.M. Time difference of arrival dilution of precision and applications. IEEE Trans. Signal Process. 1999, 47, 521-523. [CrossRef]

27. Yang, X.; Brock, R. State University of New York, 2000. RDOP Surface for GPS Relative Positioning. U.S. Patent 6,057,800, 2 May 2000.

28. Hofmann-Wellenhof, B.; Lichtenegger, H.; Collins, J. Global Positioning System: Theory and Practice, 1st ed.; Springer: Wien, Austria; New York, NY, USA, 1992; ISBN 978-3-7091-5126-6.

29. Teunissen, P.J. Influence of ambiguity precision on the success rate of GNSS integer ambiguity bootstrapping. J. Geodesy 2007, 81, 351-358. [CrossRef]

30. Böhm, J.; Möller, G.; Schindelegger, M.; Pain, G.; Weber, R. Development of an improved empirical model for slant delays in the troposphere (GPT2w). GPS Solut. 2015, 19, 433-441. [CrossRef] 
31. Yang, Y.; Xu, Y.; Li, J.; Yang, C. Progress and performance evaluation of BeiDou global navigation satellite system: Data analysis based on BDS-3 demonstration system. Sci. China-Earth Sci. 2018, 61, 614-624. [CrossRef]

32. Zhang, Z.; Li, B.; Nie, L.; Wei, C.; Jia, S.; Jiang, S. Initial assessment of BeiDou-3 global navigation satellite system: Signal quality, RTK and PPP. GPS Solut. 2019, 23, 111. [CrossRef]

33. Wu, M.; Liu, W.; Wang, W.; Zhang, X. Differential inter-system biases estimation and initial assessment of instantaneous tightly combined RTK with BDS-3, GPS, and Galileo. Remote Sens. 2019, 11, 1430. [CrossRef]

34. Tian, Y.; Sui, L.; Xiao, G.; Zhao, D. Analysis of Galileo/BDS/GPS signals and RTK performance. GPS Solut. 2019, 23, 1-16. [CrossRef]

35. Li, B.; Feng, Y.; Shen, Y.; Wang, C. Geometry-specified troposphere decorrelation for subcentimeter real-time kinematic solutions over long baselines. J. Geophys. Res. Solid Earth 2010, 115, B11404. [CrossRef] 